\title{
Failed back surgery syndrome: review and new hypotheses
}

\author{
This article was published in the following Dove Press journal: \\ Journal of Pain Research \\ 12 January 2016 \\ Number of times this article has been viewed
}

\author{
Bruno Bordoni ${ }^{1,2}$ \\ Fabiola Marelli² \\ 'Department of Cardiology, \\ Foundation Don Carlo Gnocchi, \\ IRCCS, Institute of Hospitalization \\ and Care, S Maria Nascente, Milan, \\ ${ }^{2}$ School CRESO, Osteopathic Centre \\ for Research and Studies, Falconara \\ Marittima, Ancona, Italy
}

\begin{abstract}
Failed back surgery syndrome (FBSS) is a term used to define an unsatisfactory outcome of a patient who underwent spinal surgery, irrespective of type or intervention area, with persistent pain in the lumbosacral region with or without it radiating to the leg. The possible reasons and risk factors that would lead to FBSS can be found in distinct phases: in problems already present in the patient before a surgical approach, such as spinal instability, during surgery (for example, from a mistake by the surgeon), or in the postintervention phase in relation to infections or biomechanical alterations. This article reviews the current literature on FBSS and tries to give a new hypothesis to understand the reasons for this clinical problem. The dysfunction of the diaphragm muscle is a component that is not taken into account when trying to understand the reasons for this syndrome, as there is no existing literature on the subject. The diaphragm is involved in chronic lower back and sacroiliac pain and plays an important role in the management of pain perception.
\end{abstract}

Keywords: diaphragm, fascia, chronic pain, pain, spine FBSS

\section{Introduction}

Failed back surgery syndrome (FBSS) is a term used to define an unsatisfactory outcome of a patient who underwent spinal surgery, irrespective of type or intervention area, with persistent pain in the lumbosacral region with or without it radiating to the leg. ${ }^{1,2}$ Pain may appear after surgery and persist despite the intervention for up to 3 months, with chronic consequences. ${ }^{2}$ There are other definitions for the same disorder such as postlumbar surgery syndrome, postlaminectomy syndrome, failed back syndrome, and postoperative persistent syndrome. ${ }^{3,4}$ In the text studied, the percentage of pain detected after spinal surgery varies ranging from a low of $5 \%$ to a high of $74.6 \%$, and the percentage of need for re-operation ranging from $13.4 \%$ to $35 \%{ }^{2,5-7}$ According to some authors, the percentages are not consistent with the type of intervention. If the patient has undergone a minimally invasive intervention, for example, a microdiscectomy, it does not mean that the patient will present with more minor symptoms than if a major surgical intervention had taken place, for example, fusion of the vertebrae. ${ }^{2,5-7}$ Other authors report that the type of surgical approach may influence the response rate to FBSS in favor of microsurgery. ${ }^{8}$ At present there are no surgical strategies able to prevent the instances of FBSS.

\section{Risk factors}

Literature identifies the possible risk factors leading to such chronic syndrome, divided into pre- and postintervention risk, and risks occurring during the surgical procedure. The patient may present with spinal instability prior to surgery, or in proceeding to surgery, 
there may be anomalies shown in the clinical images without good clinical correlation. ${ }^{7}$ There is a nonexhaustive relationship between the surgeon and the patient's response to surgery. ${ }^{8}$ Another preintervention risk factor is linked to the cause that leads to disease or spinal radiculopathy arising from metabolic problems (diabetes), vascular, viral, previous trauma, and tumors. ${ }^{7}$ It is acknowledged that psychosocial factors also have an effect on the presence of FBSS. Patients who demonstrate a psychologically altered state, such as depression, anxiety, somatization, and hypochondria, are more prone to unsatisfactory surgical outcomes. ${ }^{2,5,7}$

Risk factors during the surgical procedure that would affect response rate of FBSS are especially related to surgeon error, such as an incorrect vertebral localization of the problem or a partially completed operation (eg, an inadequate decompression). ${ }^{7}$ The surgical procedure itself (vertebroplasty or kyphoplasty) could create vertebral fractures, independent from intervention of the surgeon. ${ }^{7}$

Oversights by the surgeon, such as an aggressive decompression could lead to spinal instability, or a nonperfect positioning of the means of synthesis, and an erroneous clinical decision of the requirement for surgery. ${ }^{2,6-9}$ The unfavorable factors on encountering FBSS after surgery are manifold, such as postoperative complications in the presence of hematomas or infections. ${ }^{7}$ The presence of pseudarthrosis after surgical fusion and encountering epidural fibrosis alter the function and nutrition to the root of nerve. ${ }^{1,2,5,7,8}$

Anatomic and biomechanical intervention-related alterations may lead to instability of the spine, sacroiliac dysfunction, and foraminal compressions or overburden an area of the vertebra or other vertebral areas and predispose the patient to further spinal pathology, similar to those that led the patient to have surgery. ${ }^{2,5-8}$

Another postoperative factor is changes in the myofascial system connected to the vertebral area of the surgery, in particular to the paraspinal muscles in spasm or hypotrophy, which can cause referred pain that is difficult to distinguish from real spinal radiculopathy. ${ }^{7}$ The same biomechanical alterations lead to proprioceptive disturbances of other muscles, like the multifidus, lumbar rotators and the transverse abdominis, which, similar to a vicious circle, will cause pain in the lumbar region. ${ }^{7,10}$ Currently, there is no "Gold" standard in the cure and treatment of FBSS. ${ }^{11}$

\section{The diaphragm in the context of FBSS}

One component that is not even considered when trying to understand the reasons for FBSS is dysfunction of the diaphragm muscle, which is not referred to in the literature. The diaphragm is involved in chronic lower back and sacroiliac pain and plays an important role in the management of pain perception.

The respiratory diaphragm muscle is innervated by the phrenic nerve (C3-C5) and the vagus nerve (cranial nerve X); the first receives pulses from groups of medullary neurons of the Pre-Bötzinger complex and neurons of the parafacial retrotrapezoid complex, which in turn receive orders over retroambiguus from the core of the bulb, although the mechanisms that underlie these links are not completely clear. ${ }^{12}$ The vagus nerve is part of the autonomous parasympathetic system originating from the nucleus ambiguous medulla oblongata. ${ }^{13}$ The phrenic nerve and the vagus nerve are anastomosed at the cervical level. ${ }^{12}$

The diaphragm muscle is the main respiratory muscle able to influence the act of breathing through its contraction. ${ }^{14}$

The diaphragm works in various processes such as expectoration of sputum by coughing, the action of vomiting, defecation, and swallowing. ${ }^{15}$ It facilitates the venous and lymphatic return, allows the viscera above and below the diaphragm to function properly, and works with the urination function. ${ }^{12,15}$

The diaphragm is a muscle structure vital for posture, its maintenance and changes of body position. ${ }^{16,17}$

\section{Dysfunction of the diaphragm}

Dysfunction of the diaphragm is an important factor and recognized as being one of the causes of low back and sacroiliac joint pain. People who suffer from low back and sacroiliac joint pain often have early fatigue of the diaphragm muscle, altered and diminished respiratory excursion as well as inadequate proprioceptive function. ${ }^{18-22}$ The diaphragm dynamically stabilizes the lumbar spine. It lowers on inhalation, stabilizing the abdominal pressure, together with the lower ribs, which have moved downwards, with a larger movement for its ventral size compared to the dorsal area. ${ }^{22}$ In people with chronic lumbar problems, the diaphragm remains higher and more flattened, with the ventral portion moving a smaller percentage. There is a close relationship between a reduction in the movement of the diaphragm and the intensity of pain in people who suffer from low back pain. ${ }^{22}$ When the lower limbs are called upon to work, the diaphragm is activated to stabilize the spine and allow the movement required; in people with chronic pain this happens to a lesser extent. The ribs do not drop and do not allow the diaphragm to have a fixed point to lower to; there is a minor reduction in the diaphragm dome with reduced ability to manage the intra-abdominal pressure, ultimately causing lumbosacral instability. ${ }^{22}$ 
This biomechanical alteration is also found in trauma to the spinal column, for example, resulting from an accidental fall that alters the lumbar movement changing the pattern of diaphragm activation. ${ }^{22}$

When the diaphragm is not working correctly, its proprioceptive ability is reduced, further slowing down its stabilizing function on the lumbar region. ${ }^{20}$ This is true even if other muscles that contribute to the stabilization of the back do not work properly (as the paraspinal muscles, the abdominal muscles and the transversus abdominis, the quadratus lumborum and the gluteus medius), which may interfere negatively on the proprioceptive function of the diaphragm..$^{20,23,24}$

Another report to be considered in further understanding the complex functions of the diaphragm muscle as a stabilizer of the lower back is the thoracolumbar fascia (TLF). The TLF is defined by Willard et al as:

... a complex myofascial and aponeurotic girdle surrounding the torso. On the posterior body wall, the central point of this girdling structure is the thoracolumbar fascia (TLF),

a blending of aponeurotic and fascial planes that forms the retinaculum around the Paraspinal muscles of the lower

back and sacral region. ${ }^{25}$

The lateral and medial pillars of the diaphragm are in close connection with the lumbar vertebrae and the lower ribs, and in such a way that the arched pillars of the diaphragm act as a bridge between the TLF posteriorly and the transversalis fascia anteriorly. ${ }^{12}$ The TLF system allows for the tensions generated by movement and breathing along the spine of the back to be conveyed correctly, creating synergy with the lowering of the diaphragm, a sort of "sleeve" that surrounds the lumbar vertebrae, allowing for stabilisation. ${ }^{12,26,27}$ The thoracolumbar spinal fascial tissue damaged by surgery will not have the same elastic and proprioceptive capacity, compared to an intact tissue, which causes mechanical malfunctions; a TLF in difficulty leads to instability of the spine. ${ }^{28-30}$ Probably, the functional loss of this fascial system may disturb the work of the diaphragm, causing a cascade of pathological events such as pain and biomechanical alterations in the lumbosacral region.

It can be strongly hypothesized that a dysfunction of the diaphragm muscle as a stabilizer to the lumbosacral column is one of the causes leading to FBSS that is caused by factors preceding surgery or following spinal surgery. A function of the diaphragm that could be disturbed in FBSS is its analgesic action, this dysfunction can become one of the causes of chronic pain.

\section{Pain, emotions, and the breath}

Chronic pain that characterizes FBSS may have different origins, probably due to the varied patient histories and the type of surgical intervention. Pain can be defined as nociceptive when it is a painful stimulus that triggers the inflammatory reaction, when the stimulus that causes it is derived from immune or biochemical alterations as in the case of injury and neuropathic pain when there is an injury to the nervous system. ${ }^{31}$ Constant stimulation of the nociceptive system forces a change to the plastic nervous, peripheral and central structures, constituting what is called a central sensitization. ${ }^{31}$ This event is manifested as a reduction of the threshold of spinal neurons (allodynia), increased stimulus response becomes active and prolonged even when the stimulus causing it has ceased (hyperalgesia), and a modified response from other nondamaged tissues that cause the pain (secondary hyperalgesia). ${ }^{31}$ The information may arise from the facet joints, the spinal disc, and soft tissue such as ligaments and myofascial tissue, or more tissues simultaneously, creating a multitude of information that overlap the different definitions of pain. ${ }^{32-35}$ According to recent research, the sympathetic nervous system is overstimulated in FBSS syndrome and this autonomic dysfunction may contribute to the chronicity of pain in this population of patients. $^{36}$

The perception of pain is diminished if the breath is held following a deep inhalation, a condition in which the diaphragm is lowered. ${ }^{37}$ This event appears to reflect the involvement of baroreceptors. In doing so, the respiratory systolic pressure increases with a decrease in the cardiac frequency. ${ }^{37}$ We know that when the baroreceptor sites in the carotid body and in the area of the aortic arch in the adventitia of the vessels are naturally stimulated by the cardiac cycle, in particular by the systole, the nociceptive stimulus is lessened by the activation of baroceptors. ${ }^{38}$

The intervention baroreceptor also affects muscle tone, as it decreases the activity of the sympathetic nervous system, reducing its contractile tone. ${ }^{38}$ The reduction in pain perception seems to be greater if the subject is aware of the pain itself. ${ }^{38}$ Chronic and acute pain can alter the baroreceptor function and consequently damage the regulatory function of the cardiovascular system; this in the long run will lead to a higher risk of mortality and morbidity. ${ }^{39}$

The baroreceptors are structures that are activated if the vessel is stretched by the passing blood. ${ }^{40}$ The afferents that branch are collected from the nucleus of the solitary tract (NTS), which modulates the intervention of the efferent vagal system and the efferent inhibitory sympathetic nerve in the spinal cord near the nucleus ambiguous, the dorsal motor nucleus, and the rostral ventrolateral area of the medulla oblongata. ${ }^{40}$ Baroreceptor afferents affect different areas of the central nervous system, with a generalized inhibitory effect. ${ }^{40}$ 
The NTS connects with the reticular formation, from which information is sent to the anterior, lateral and medial prefrontal and insula and the anterior cingulate cortex; even the thalamus, hypothalamus, and periaqueductal gray area receive signals from NTS baroreceptors. ${ }^{40}$

There is a close relationship between emotions, breathing, and the intervention of baroreceptors. ${ }^{40}$ The emotional experience influences the response to pain, because the pain response is not simply a neural process started by nociceptive afferents. ${ }^{38}$ Emotional states such as anxiety or depression and psychiatric disorders are able to negatively alter the baroreceptors' response. ${ }^{41}$ Stress that causes anxiety and/or depression will cause impaired function of the diaphragm. ${ }^{42}$ Modifications to the emotional state cause an increase in the perception of pain. ${ }^{43}$

It can be said that the diaphragm has an influence on baroreceptors and the perception of pain and vice versa.

The diaphragm and its movements change the body pressure, in that the diaphragm facilitates the venous and lymphatic return upwards. ${ }^{12}$ This modulation in pressure influences the re-distribution of blood. ${ }^{44}$ It is very likely that this determines the baroreceptor, response and a reduction in pain perception; however, there are still no scientific texts to support this assertion. Recent scientific evidence highlights the ability of the vagus nerve to carry painful afferents, especially in respect of visceral pain. ${ }^{45}$ It is known that the NTS stimulates the vagus nerve, and it can be assumed that a physiological function of the diaphragm muscle can somehow reduce nociceptive stimulation of the vagus nerve from the central nervous system, or through pressure and proper functions of the viscera from lowering the diaphragm that does not irritate the tenth cranial nerve, therefore acting peripherally. ${ }^{46}$ There is no current scientific evidence to confirm this thought.

An incorrect diaphragm position as in chronic low back pathologies could lead to inadequate stimulation of baroreceptors and incorrect function of the same; this could lead to a heightened sensibility to a greater feeling of pain. The same diaphragm can be a source of pain afferents probably due to the phrenic nerve, a mixed nerve that carries motor and sensory information, sharing information with the spinal trigeminal nucleus. ${ }^{12,47,48}$ The spinal trigeminal nucleus has a connection with the NTS and it could be hypothesized that this connection is the cause of the pain coming from the diaphragm. ${ }^{49}$

The diaphragm has a phrenic center, consisting of a strong "V" shaped connective component with a variable percentage in respect to the amount of contractile tissue. ${ }^{50}$ The fascial system is richly innervated by proprioceptors, which can become a source of painful afferents that can transform into nociceptors. ${ }^{26}$ The crural and connective tissue areas are populated by proprioceptors and it can be assumed that an alteration to the position and function of the respiratory muscle creates a state of irritability of these proprioceptors and subsequent presence of painful afferents. ${ }^{12}$ It can be assumed that if the position of the diaphragm is not physiological, the phrenic nerve is retracted or irritated in different ways, causing nociceptive afferents, in the same way as for a peripheral nerve irritation from the surrounding tissues that it crosses. ${ }^{51,52}$

If scientific research were to prove that the diaphragm muscle plays an important role in FBSS, the therapeutic approach might provide an additional step toward improving the clinical condition and quality of life in this patient population. We await further studies.

\section{Conclusion}

A component that is not even considered when trying to understand the causes that lead to FBSS is the dysfunction of the diaphragm muscle, such that texts in literature do not mention the subject. The diaphragm is involved in chronic lower back and sacroiliac pain and plays an important part in the management of pain perception. Its dysfunction due to positional alterations could be one of the major underlying causes of chronic pain in this patient population. This is because the diaphragm dysfunction would lead to alterations in the biomechanics of the lumbar spine, with less proprioceptive abilities, less movement of the vertebrae, and reduction of functional collaboration of tissues that are involved in the proper functioning of the lumbar area; or less stabilization, or it provides less stimulation of baroreceptors by the diaphragm and an alteration in the perception of pain. In conclusion, the diaphragm itself could be a source of pain, due to the change of its proprioceptors or irritation of the phrenic nerve and the vagus nerve. If scientific research were to prove that the diaphragm muscle plays an important role in FBSS, the therapeutic approach might provide an additional step toward improving the clinical condition and quality of life in this patient population.

\section{Disclosure}

The authors report no conflicts of interest in this work.

\section{References}

1. Avellanal M, Diaz-Reganon G, Orts A, Soto S. One-year results of an algorithmic approach to managing failed back surgery syndrome. Pain Res Manag. 2014;19(6):313-316.

2. Hussain A, Erdek M. Interventional pain management for failed back surgery syndrome. Pain Pract. 2014;14(1):64-78. 
3. Rigoard P, Desai MJ, Taylor RS. Failed back surgery syndrome: what's in a name? A proposal to replace "FBSS" by "POPS." Neurochirurgie. 2015;61 Suppl 1:S16-S21.

4. Chan CW, Peng P. Failed back surgery syndrome. Pain Med. 2011;12(4): 577-606.

5. Choi HS, Chi EH, Kim MR, et al. Demographic characteristics and medical service use of failed back surgery syndrome patients at an integrated treatment hospital focusing on complementary and alternative medicine: a retrospective review of electronic medical records. Evid Based Complement Alternat Med. 2014;2014:714389.

6. Bodiu A. Diagnosis and operatory treatment of the patients with failed back surgery caused by herniated disk relapse. J Med Life. 2014;7(4): 533-537.

7. Shapiro C. The failed back surgery syndrome: pitfalls surrounding evaluation and treatment. Phys Med Rehabil Clin N Am. 2014;25(2) 319-340.

8. Harper WL, Schmidt WK, Kubat NJ, Isenberg RA. An open-label pilot study of pulsed electromagnetic field therapy in the treatment of failed back surgery syndrome pain. Int Med Case Rep J. 2014;8:13-22.

9. Romero-Vargas S, Obil-Chavarria C, Zárate-Kalfopolus B, RosalesOlivares LM, Alpizar-Aguirre A, Reyes-Sánchez AA. Profile of the patient with failed back surgery syndrome in the National Institute of Rehabilitation. Comparative analysis. Cir Cir. 2015;83(2):117-123.

10. Ramsook RR, Malanga GA. Myofascial low back pain. Curr Pain Headache Rep. 2012;16(5):423-432.

11. Desai MJ, Nava A, Rigoard P, Shah B, Taylor RS. Optimal medical, rehabilitation and behavioral management in the setting of failed back surgery syndrome. Neurochirurgie. 2015;61 Suppl 1:S66-S76.

12. Bordoni B, Zanier E. Anatomic connections of the diaphragm: influence of respiration on the body system. J Multidiscip Healthc. 2013;6 281-291.

13. Shen MJ, Zipes DP. Role of the autonomic nervous system in modulating cardiac arrhythmias. Circ Res. 2014;114(6):1004-1021.

14. Bordoni B, Zanier E. The continuity of the body: hypothesis of treatment of the five diaphragms. J Altern Complement Med. 2015;21(4): 237-242.

15. Chaitow L, Gilbert C, Morrison D. Recognizing and Treating Breathing Disorders. A Multidisciplinary Approach. Oxford: Churchill Livingstone; 2013.

16. Hamaoui A, Hudson AL, Laviolette L, Nierat MC, Do MC, Similowski T. Postural disturbances resulting from unilateral and bilateral diaphragm contractions: a phrenic nerve stimulation study. J Appl Physiol (1985). 2014;117(8):825-832.

17. Bradley H, Esformes J. Breathing pattern disorders and functional movement. Int J Sports Phys Ther. 2014;9(1):28-39.

18. Janssens L, Brumagne S, McConnell AK, Hermans G, Troosters T, Gayan-Ramirez G. Greater diaphragm fatigability in individuals with recurrent low back pain. Respir Physiol Neurobiol. 2013;188(2) $119-123$

19. Kolar P, Sulc J, Kyncl M, et al. Postural function of the diaphragm in persons with and without chronic low back pain. J Orthop Sports Phys Ther. 2012;42(4):352-362.

20. Janssens L, McConnell AK, Pijnenburg M, et al. Inspiratory muscle training affects proprioceptive use and low back pain. Med Sci Sports Exerc. 2015;47(1):12-19.

21. O'Sullivan PB, Beales DJ. Changes in pelvic floor and diaphragm kinematics and respiratory patterns in subjects with sacroiliac joint pain following a motor learning intervention: a case series. Man Ther 2007;12(3):209-218.

22. Vostatek P, Novák D, Rychnovský T, Rychnovská S. Diaphragm postural function analysis using magnetic resonance imaging. PLoS One. 2013;8(3):e56724.

23. Finn C. Rehabilitation of low back pain in golfers: from diagnosis to return to sport. Sports Health. 2013;5(4):313-319.

24. Cooper NA, Scavo KM, Strickland KJ, et al. Prevalence of gluteus medius weakness in people with chronic low back pain compared to healthy controls. Eur Spine J. Epub 2015 May 26.
25. Willard FH, Vleeming A, Schuenke MD, Danneels L, Schleip R. The thoracolumbar fascia: anatomy, function and clinical considerations. J Anat. 2012;221(6):507-536.

26. Bordoni B, Zanier E. Clinical and symptomatological reflections: the fascial system. J Multidiscip Healthc. 2014;7:401-411.

27. Vleeming A, Schuenke MD, Danneels L, Willard FH. The functional coupling of the deep abdominal and paraspinal muscles: the effects of simulated paraspinal muscle contraction on force transfer to the middle and posterior layer of the thoracolumbar fascia. $J$ Anat. 2014;225(4): $447-462$.

28. Bordoni B, Zanier E. Skin, fascias, and scars: symptoms and systemic connections. J Multidiscip Healthc. 2013;7:11-24.

29. Langevin HM, Fox JR, Koptiuch C, et al. Reduced thoracolumbar fascia shear strain in human chronic low back pain. BMC Musculoskelet Disord. 2011;12:203.

30. Yan Y, Xu R, Zou T. Is thoracolumbar fascia injury the cause of residual back pain after percutaneous vertebroplasty? A prospective cohort study. Osteoporos Int. 2015;26(3):1119-1124.

31. Woolf CJ. Central sensitization: implications for the diagnosis and treatment of pain. Pain. 2011;152(3 Suppl):S2-S15.

32. Cincu R, Lorente Fde A, Gomez J, Eiras J, Agrawal A. A 10-year follow-up of transpedicular screw fixation and intervertebral autogenous posterior iliac crest bone graft or intervertebral B-Twin system in failed back surgery syndrome. Asian J Neurosurg. 2015;10(2):75-82.

33. Mertens P, Blond S, David R, Rigoard P. Anatomy, physiology and neurobiology of the nociception: a focus on low back pain (part A). Neurochirurgie. 2015;61 Suppl 1:S22-S34.

34. Rigoard P, Blond S, David R, Mertens P. Pathophysiological characterisation of back pain generators in failed back surgery syndrome (part B). Neurochirurgie. 2015;61 Suppl 1:S35-S44.

35. Blond S, Mertens P, David R, Roulaud M, Rigoard P. From "mechanical" to "neuropathic" back pain concept in FBSS patients. A systematic review based on factors leading to the chronification of pain (part C). Neurochirurgie. 2015;61 Suppl 1:S45-S56.

36. El-Badawy MA, El Mikkawy DM. Sympathetic dysfunction in patients with chronic low back pain and failed back surgery syndrome. Clin J Pain. Epub 2015 May 8.

37. Reyes Del Paso GA, Muñoz Ladrón de Guevara C, Montoro CI. Breath-holding during exhalation as a simple manipulation to reduce pain perception. Pain Med. Epub 2015 Apr 30.

38. Gray MA, Minati L, Paoletti G, Critchley HD. Baroreceptor activation attenuates attentional effects on pain-evoked potentials. Pain. 2010;151(3):853-861.

39. Nielsen R, Nikolajsen L, Krøner K, et al. Pre-operative baroreflex sensitivity and efferent cardiac parasympathetic activity are correlated with post-operative pain. Acta Anaesthesiol Scand. 2015;59(4):475-485.

40. Reyes del Paso GA, Montoro C, Muñóz Ladrón de Guevara C, Duschek S, Jennings JR. The effect of baroreceptor stimulation on pain perception depends on the elicitation of the reflex cardiovascular response: evidence of the interplay between the two branches of the baroreceptor system. Biol Psychol. 2014;101:82-90.

41. Duschek S, Werner NS, Reyes Del Paso GA. The behavioral impact of baroreflex function: a review. Psychophysiology. 2013;50(12): $1183-1193$.

42. Hagman C, Janson C, Emtner M. Breathing retraining - a five-year follow-up of patients with dysfunctional breathing. Respir Med. 2011;105(8):1153-1159.

43. Vietri J, Otsubo T, Montgomery W, Tsuji T, Harada E. The incremental burden of pain in patients with depression: results of a Japanese survey. BMC Psychiatry. 2015;15:104.

44. Petersen LG, Carlsen JF, Nielsen MB, Damgaard M, Secher NH. The hydrostatic pressure indifference point underestimates orthostatic redistribution of blood in humans. J Appl Physiol (1985). 2014;116(7): 730-735.

45. Chen SL, Wu XY, Cao ZJ, et al. Subdiaphragmatic vagal afferent nerves modulate visceral pain. Am J Physiol Gastrointest Liver Physiol. 2008;294(6):G1441-G1449. 
46. Morton D, Callister R. Exercise-related transient abdominal pain (ETAP). Sports Med. 2015;45(1):23-35.

47. Blows WT. Diaphragmatic cramp as a possible cause of noncardiac chest pain and referred mandibular pain. J Neurosci Nurs. 1999;31(3): 187-190.

48. Wolf SG. Diaphragmatic spasm: a neglected cause of dyspnoea and chest pain. Integr Physiol Behav Sci. 1994;29(1):74-76.

49. Maniyar FH, Sprenger T, Schankin C, Goadsby PJ. The origin of nausea in migraine - a PET study. J Headache Pain. 2014;15:84.
50. du Plessis M, Ramai D, Shah S, Holland JD, Tubbs RS, Loukas M. The clinical anatomy of the musculotendinous part of the diaphragm. Surg Radiol Anat. 2015;37(9):1013-1020.

51. Rowe PC, Fontaine KR, Violand RL. Neuromuscular strain as a contributor to cognitive and other symptoms in chronic fatigue syndrome: hypothesis and conceptual model. Front Physiol. 2013;4:115.

52. Rickett T, Connell S, Bastijanic J, Hegde S, Shi R. Functional and mechanical evaluation of nerve stretch injury. J Med Syst. 2011;35(5): 787-793.

\section{Publish your work in this journal}

The Journal of Pain Research is an international, peer-reviewed, open access, online journal that welcomes laboratory and clinical findings in the fields of pain research and the prevention and management of pain. Original research, reviews, symposium reports, hypothesis formation and commentaries are all considered for publication.

\section{Dovepress}

The manuscript management system is completely online and includes a very quick and fair peer-review system, which is all easy to use. Visit http://www.dovepress.com/testimonials.php to read real quotes from published authors. 